\title{
Ingreso de psittacidos al centro de atención de fauna (CAV-CVS), durante los años 2007-2009
}

\author{
Entrance of parrots to the center of attention of wildlife (CAV- \\ CVS), during the years 2007-2009
}

\author{
Juan Carrascal V, ${ }^{1 *}$ M.Sc, Julio Chacón $\mathrm{P}_{1}{ }^{2}$ MVZ, Vivian Ochoa $\mathrm{C}_{1}{ }^{3} \mathrm{MVZ}$.
}

\begin{abstract}
${ }^{1}$ Universidad de Córdoba, Facultad de Medicina Veterinaria y Zootecnia, Departamento de Ciencias Pecuarias. Montería, Colombia. ${ }^{2}$ Universidad de Córdoba, Facultad de Ciencias Básicas e Ingenierías. ${ }^{3}$ Corporación Autónoma Regional de los Valles del Sinú y San Jorge - CVS. *Correspondencia: jcvelasque@yahoo.com.
\end{abstract}

Recibido: Septiembre de 2010; Aceptado: Diciembre de 2011.

\section{RESUMEN}

Objetivo. En este estudio se determinó la dinámica de ingresos de la familia Psittacidae al Centro de Atención y Valoración de Fauna Silvestre (CAV - CVS). Materiales y métodos. Se tomaron datos obtenidos desde el año 2007 hasta el año 2009, a los cuales se implementó análisis de frecuencias para las variables especies, tipo de ingreso y tiempo (meses y años), para la variable meses se aplicó un análisis de diferencia de medias y se presentó la relación ingreso de las especies versus los meses del año, por medio de un stripplot. Resultados. La familia Psittacidae presentó el $58 \%$ del total de aves que ingresaron al CAV producto de decomisos preventivos realizados en mercados públicos y vías del departamento de Córdoba. La especie con mayor frecuencia de ingresos fue Brotogeris jugularis, con $73.99 \%$ del total de individuos, siendo la especie que obtuvo los mayores ingresos durante todo el año. Asimismo, se encontró diferencias significativas para el ingreso por meses $\left(X^{2}=\right.$ $26.785, \mathrm{gl}=11, \mathrm{p}$-valor $=0.004951)$. Conclusiones. Se logró determinar que la época seca, acorde con las festividades en el departamento, presentó los valores más altos de ingresos.

Palabras clave: Brotogeris jugularis, Colombia, fauna, Psittacidae (Fuente: $C A B$ ).

\section{ABSTRACT}

Objetive. The entrance dynamics of family Psittacidae to the Wildlife Attention and Valuation Center (CAV - CVS) was determined. Materials and methods. Data were obtained between 2007 to 2009. A frequency analysis for the variables species, entrance type and time (months and years) was implemented. For the variable months an analysis of mean difference was applied and the relationship entrance of the species versus the months of the year by means of a stripplot was presented. Results. The Family Psittacidae presented 58\% of the total of birds that entered the CAV-CVS, samples collected from public markets and highways of the department of Córdoba. The species with more entrance frequency was Brotogeris jugularis, with $73.99 \%$ of the total of individuals, being that specie the one that obtained higher entrances throughout the year. Significant differences in incoming samples per month $\left(X^{2}=26.785, d f=11, p=0.004951\right)$, were also detected. Conclusions. The dry season, confluent with festivities in the department, presented the highest values of incomings to the center.

Key words: Brotogeris jugularis, Colombia, fauna, Psittacidae (Source: CAB). 


\section{INTRODUCCIÓN}

Durante un extenso periodo de la historia colombiana la fauna silvestre ha sido objeto de libre uso y aprovechamiento; una de las principales consecuencias de este accionar es la declinación de las poblaciones de algunas especies animales (1). La enorme oferta de biodiversidad ha convertido también al país en un centro importante del comercio ilegal de vida silvestre y pese a la legislación existente y a las medidas adoptadas hasta ahora para fomentar su uso sostenible y garantizar su protección, se asume que el volumen del tráfico ilegal sigue siendo de gran magnitud, pero debido a la naturaleza ilícita de la actividad, a las pocas cifras disponibles sobre extracción y comercialización de fauna en el país, y a los pocos recursos con que cuentan las autoridades ambientales, se carece de patrones que permitan establecer el impacto biológico para cada especie, así como el impacto sobre los ecosistemas (2). De acuerdo con los registros de decomisos adelantados en el período comprendido entre 1992 y 1999 por parte del Ministerio de Ambiente, varias especies de la fauna silvestre pertenecientes a las clases aves, mamíferos, reptiles, anfibios, gasterópodos, peces, arácnidos, crustáceos y antozoarios son objeto de tráfico ilegal (3).

En el caso de las aves, el comercio mundial afecta a un gran número de especies y representa una actividad económica importante para algunos países, como Perú, Bolivia y Mexico (4-7). Más de 2600 especies de aves han sido detectadas en el comercio internacional en los últimos 20 años, lo que representa aproximadamente el $27 \%$ de las especies descritas de este grupo de vertebrados (8). De acuerdo a estos, nace la estrategia nacional para la conservación de las aves de Colombia liderada por el Instituto Alexander von Humboldt, como una de sus metas la protección de especies amenazadas (9). Asimismo, se conoce que las aves mayormente decomisadas por las autoridades ambientales se encuentran representados en su gran mayoría por especímenes de la familia Psittacidae (10). A pesar de los datos registrados, se desconocen las cantidades y el por qué la gente anhela tener a los psitácidos como mascotas. Se demuestra que existe una alta demanda nacional para la tenencia de especies de psitácidos como mascotas a pesar de ser considerada una actividad ilegal (11). Sin embargo, dicha información sobre el tráfico en las Corporaciones Autónomas Regionales CAR's en general no son divulgadas, impidiéndole al Ministerio de Ambiente Vivienda y Desarrollo (MAVDT) generar las estadísticas para un conocimiento preciso del tráfico de especies en
Colombia (2) en especial al grupo de Psitácidos, el cual es considerado el más amenazado dentro de las aves colombianas (12). De acuerdo a lo anterior, en este trabajo se evaluó el ingreso de la familia Psittacidae al Centro de Atención y Valoración de Fauna Silvestre (CAV - CVS), teniendo en cuenta la naturaleza taxonómica, tipo de ingreso (Decomiso Preventivo y Entrega Voluntaria) y tiempo (años y meses).

\section{MATERIALES Y MÉTODOS}

Sitio de estudio. Este trabajo se realizó durante el periodo 2007-2009 en las instalaciones del centro de atención y fauna silvestre CAVCVS ubicado en el municipio de Montería, Departamento de Córdoba (08048'13.976" N y 75050'41.755" W).

Análisis de los datos. Este estudio presenta un análisis retrospectivo del ingreso de Psitaciformes al CAV-CVS. La información fue filtrada a partir de las bases de datos de ingresos de fauna al centro, agrupando la información de acuerdo a su naturaleza taxonómica, tipo de ingreso (Decomiso Preventivo y Entrega Voluntaria) y tiempo (años y meses), a estos datos se les aplicó un análisis de frecuencias. Para determinar si los datos se comportan normalmente se realizó el test de normalidad de Shapiro-Wilk, para luego realizar el test de Kruskal-Wallis con el fin de determinar si existía diferencia entre las medias entre los meses. Por otra parte, se realizó una grafica de puntos (stripplot) para comparar el ingreso de las especies según los meses. Estos análisis se realizaron con ayuda del software R 2.10.1.

\section{RESULTADOS}

Al centro de atención de fauna silvestre CAVCVS en el periodo comprendido entre enero de 2007 y diciembre de 2009 ingresaron un total de 1111 individuos pertenecientes a 12 especies del orden Psitaciformes (Tabla 1 ), equivalentes al $53.98 \%$ de las aves que ingresaron al CAV durante el periodo de estudio. Cabe resaltar que solo un individuo no fue posible identificar a nivel específico por las condiciones de ingreso. Por su parte, la especie que presentó el porcentaje más alto de ingreso fue el perico (Brotogeris jugularis) con un $73.99 \%$ del total de individuos, seguida de la especie Aratinga pertinax (Cotorra) con un $12.87 \%$ (Figura 1 ).

De acuerdo al tipo de ingreso, se encontró que los Psitácidos que ingresaron al CAV-CVS fue 
Tabla 1. Listado de especies de Psitácidos que ingresaron al CAV-CVS durante el periodo comprendido entre enero de 2007 y diciembre de 2009.

\begin{tabular}{|c|c|c|}
\hline Género & Nombre Científico & Nombre Común \\
\hline \multirow[t]{5}{*}{ Amazona } & Amazona amazonica & Lora cari amarilla \\
\hline & Amazona autumnalis & Lora moña roja \\
\hline & Amazona farinosa & Lora mojosa \\
\hline & Amazona ochrocephala & Lora moña amarilla \\
\hline & Amazona sp & Lora \\
\hline \multirow[t]{3}{*}{ Ara } & Ara ararauna & $\begin{array}{l}\text { Guacamaya Vientre } \\
\text { Amarillo }\end{array}$ \\
\hline & Ara macao & Guacamaya Bandera \\
\hline & Ara severa & Cheja \\
\hline Aratinga & Aratinga pertinax & Cotorra \\
\hline Brotogeris & Brotogeris jugularis & Perico \\
\hline Forpus & Forpus conspicillatus & Cascabelito \\
\hline Pionus & Pionus menstruus & Loro cabeciazul \\
\hline
\end{tabular}

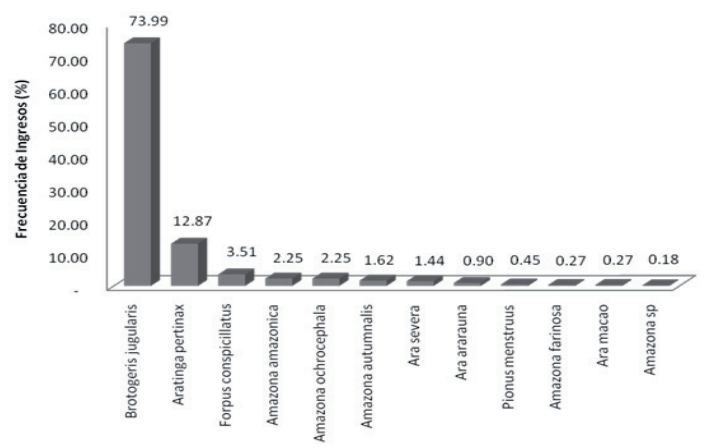

Figura 1. Frecuencia de ingreso por especies de Psitácidos al Centro de Atención y Valoración CAV-CVS entre los años 2007 y 2009.

dada mayormente por decomisos preventivos $(97.66 \%)$ realizados en los principales mercados públicos y vías carreteables del departamento de Córdoba.

El análisis de la frecuencia de ingresos por año mostró que el año 2008 obtuvo el mayor porcentaje de ingresos de Psitácidos al CAV con un $37.17 \%$ del total de individuos. Al realizar este análisis teniendo en cuenta los meses se encontró que los picos más altos de ingresos de las especies de Psitácidos al CAV-CVS se dan principalmente en los meses de febrero (20.16\%) con aumento en el mes de marzo (29.52\%), así como también en los meses de diciembre (10.98) y julio (9.9\%) (Figura. 2).

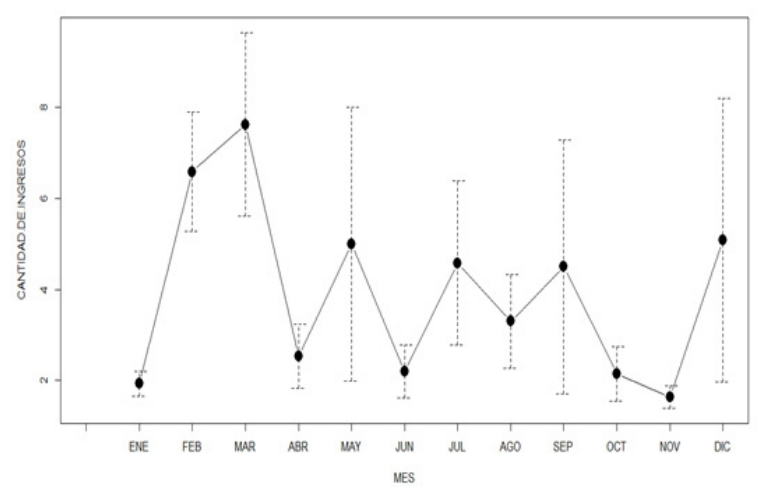

Figura 2. Variación mensual de ingresos de Psitácidos al Centro de Atención y Valoración CAV-CVS entre los años 2007 y 2009.

Se encontró que los datos se comportan normalmente al aplicar el test de Shapiro - WilK (W = 0.4114, p-valor < 2.2e-16). De acuerdo a esto, el test de Kruskal-Wallis mostró que existen diferencias significativas en el ingreso de psitácidos entre el análisis de ingresos mensuales ( $\mathrm{X} 2=26.785, \mathrm{gl}=11$, $\mathrm{p}$-valor $=0.004951)$. Al observar la gráfica de puntos para la relación ingreso de las especies por mes se puede notar que Brotogeris jugularis mostró los mayores ingresos a lo largo del año (Figura 3).

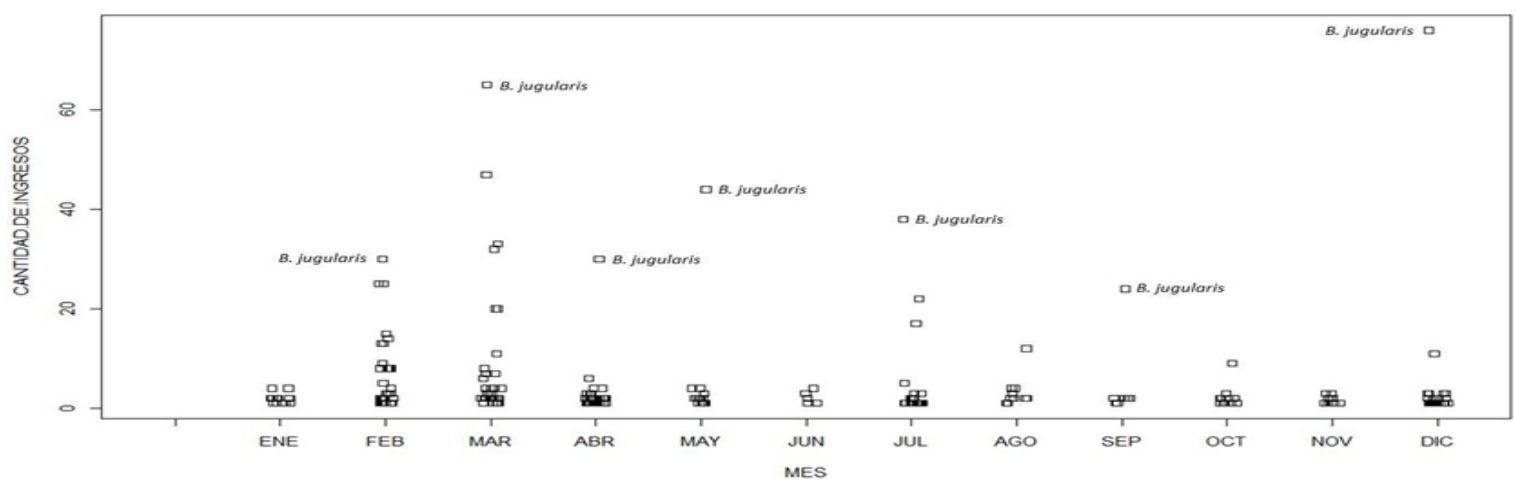

Figura 3. Diagrama de puntos para el ingresos de Psitácidos al Centro de Atención de Fauna Silvestre (CAVCVS) entre los años 2007 y 2009. 


\section{DISCUSIÓN}

Al igual que lo mostrado en las estadísticas del uso ilegal de fauna silvestre en Colombia (13) y lo reportado por la CITES, donde para los años 1994-1996 la familia Psittacidae es la más afectada por el comercio ilícito a nivel global, con el $32.2 . \%$ del total de familias objeto de tráfico ilegal (14). Este trabajo muestró que el orden Psittaciformes presenta valores superiores al $50 \%$ del total de las aves que ingresaron al CAV. Es de anotar que la mayor parte de los individuos que ingresan al centro se encuentran en estado juvenil o neonatos, extraídos de sus nidadas. Dicha extracción de nidos es un factor de mortalidad de psitácidos generalizado y biológicamente significativo (15). Para México, se conoce que la cadena captura, transporte, distribución y venta, genera mortalidades estimadas del $77 \%$ de los individuos antes de poder ser entregados al consumidor, dado al trato inhumano que se le da a los individuos (16). De acuerdo a esto, esta situación advierte el colapso de las poblaciones de psitácidos en áreas naturales (10).

Brotogeris jugularis presentó mayores decomisos durante todo el año (Figuras. 1, 3), siendo concordante con lo reportado en distintas partes del país, como en Girardot donde esta especie es comercializada ilegalmente principalmente en el mes de abril (11). De igual manera, según el Ministerio del Medio Ambiente $B$. jugularis presenta los mayores porcentajes de decomisos en el país (13). Igualmente, en otros países como Nicaragua esta especie es considerada como la de mayor comercialización por vendedores ambulantes en vías y mercados públicos, principalmente por su pequeño tamaño y fácil captura (17).

Por otro lado, el ingreso de especies pertenecientes a los géneros Ara y Amazona, las cuales son consideradas dentro del Libro Rojo de Aves de Colombia y categorizadas dentro del listado de especies de la CITES (10, 18), muestran que Córdoba hace parte del eje de trafico de estas especies en el país, siendo concordante con lo mostrado por Baptiste y Franco (19), quienes advierten en su libro "Especies focales para la conservación en el departamento de Córdoba" que todas las especies de psitacidos muestran algún grado de amenaza por la presión de la cacería ilegal y la destrucción de los hábitats.

En general, la familia Psittacidae sufre la mayor presión de cacería en época seca, la cual coincide con la época de reproducción de estas especies. Los psitacidos son capturados en las nidadas (huecos de árboles y termiteros) principalmente en estado juvenil o neonatal (20). Sumado a esto, es en estas épocas donde se da la mayor afluencia de turistas debido a las vacaciones, Semana Santa y festividades, que promueven no solo la venta de la oferta presente en los mercados, sino la extracción de más individuos para compensar la demanda de estas especies (11). Los resultados de este estudio mostraron diferencias significativas existentes entre los meses, dado por el aumento de ingresos en los meses de febrero y marzo, como se anotó anteriormente.

En conclusión, los psitácidos representan el 58\% de las aves que ingresan al Centro de Atención y Valoración (CAV - CVS), siendo la especie Brotogeris jugularis la que sufre mayor impacto por tráfico ilegal a lo largo del año. Asimismo, el tráfico ilegal de Psitácidos en el departamento de Córdoba se da principalmente en la época seca (febrero y marzo), causando disminuciones importantes en las poblaciones naturales, debido a que estos índices de decomisos concuerdan con las épocas de reproducción de estas especies. El departamento de Córdoba, se considera como un eje de tráfico a nivel nacional dado a que se dan procesos extractivos importantes, principalmente en especies con altos grados de amenaza.

\section{Agradecimientos}

Al Centro de Atención y Valoración (CAV-CVS) y a todo el personal que labora ahí, por permitir acceder a los datos de ingresos de fauna. A Roger Ayazo por su ayuda en el análisis de la información y a todas aquellas personas que de alguna u otra forma colaboraron para que este trabajo pudiera realizarse. 


\section{REFERENCIAS}

1. Lara-Vásquez, Castaño-Rivas y Jonker. Notas acerca de las guacamayas (Psittacidae: Ara) introducidas en el municipio de Medellín, Colombia. Boletín SAO 2007; 17(02): 104-110.

2. Mancera, N. y Reyes, O. Comercio de Fauna Silvestre en Colombia. Revista Facultad Nacional Agropecuaria. Medellín 2008; 61(2):4618-4645.

3. Ministerio del Medio Ambiente. Estrategia Nacional para la Prevención y Control del Tráfico Ilegal de Especies Silvestres. Colombia: Dirección general de ecosistemas; 2002.

4. Ministerio de Ambiente del Ecuador. Situación actual del tráfico ilegal de la vida silvestre. Ecuador: Dirección nacional de biodiversidad unidad de vida silvestre. Ministerio del Ambiente. 2008.

5. Naranjo E, Dirzo R, López J, Rendonvon J, Reuter A, Sosa-Nishizaki $O$ et al. Impacto de los factores antropogénicos de afectación directa a las poblaciones silves $\neg$ tres de flora y fauna, en Capital natural de México: Estado de conservación y tendencias de cambio. Conabio 2009; 2:247-276.

6. Lezama, M.; Vilchez, S.; Mayorga, M. \& Castellon, R. III Monitoreo de psitácidos estado actual y conservación. Nicaragua: Secretaría de la Convención Sobre el Comercio Internacional de Especies Amenazadas de Fauna y Flora Silvestre (CITES), Nicaragua y Corredor Biológico del Atlántico (CBA), Ministerio del Ambiente y los Recursos Naturales. 2005.

7. Ferreira S. The illegal parrot trade in the neo-tropics: the relationship between poaching and illicit pet markets. Dissertation Newark Rutgers, the State University of New Jersey, Program in Criminal Justice. 2012.

8. Guix J. C., Jover LI. y Ruiz X. Muestreos del comercio de psitácidos neotropicales en la ciudad de Barcelona, España: 1991-1996. Ararajuba 1997; 5(2):159-167.
9. Renjifo L. M., Franco H, Álvarez-López M, Álvarez R, Borja J, Botero $S$, et al. Estrategia nacional para la conservación de las aves de Colombia. Bogotá, Colombia. Instituto Alexander von Humboldt 2000.

10. Renjifo LM, Franco AM, Amaya JD, Catan GH. y López B. (eds. ). Libro rojo de aves de Colombia. Serie Libros Rojos de Especies Amenazadas de Colombia. Bogotá, D.C.: Instituto de Investigación de Recursos Biológicos Alexander von Humboldt y Ministerio del Medio Ambiente; 2002.

11. Baquero M. Dinámica de comercialización ilegal de especies de la familia Psittacidae y contexto sociocultural en las ciudades de Villavicencio, Girardot, Bogota D.C. y el Municipio del Espinal, Colombia. [Tesis de Pregrado] Bogotá, Colombia: Universidad Pontificia Javeriana, Departamento de Ecología; 2004.

12. Quevedo-Gil A. Plan de acción nacional para la conservación de los loros amenazados. Conservación Colombiana. 2006: 1.

13. Gómez M. Estadísticas del uso ilegal de fauna silvestre en Colombia. Republica de Colombia: Ministerio del Medio Ambiente. Direccion General de Ecosistemas, Grupo de Biodiversidad. [Acceso: 10 de marzo de 2010]. URL:http://www1.minambiente. gov.co/viceministerios/ambiente/dir ecosistemas/conservacion_biodiversidad/ uso_sostenible/estadisticas_trafico.pdf

14. Roda, Franco AM., Baptiste M.P., Mónera C. y Gómez D.M. Manual de identificación CITES de aves de Colombia. Serie Manuales de Identificación CITES de Colombia. Instituto de Investigación de Recursos Biológicos Alcxander von Humboldt y Ministerio de Ambiente, Vivienda y Desarrollo Territorial, Bogotá, Colombia. 2003: 352.

15. Wright $T$, Toft $E$, Enquerlin-Hoeflich J, González-Elizondo $M$, Abornoz A, Rodríguez-Ferraro $F$, et al. Nest poaching in neotropical parrots. Conservation Biology $2001 ; 15(3): 710-720$. 
16. Cantu, J. C. G., Saldana, M. E. S., Grosselet, M., and Gamez, J. S.. The Illegal Parrot Trade in Mexico: A Comprehensive Assessment. Mexico and Washington, DC: Defenders of Wildlife; 2007.

17. Herrera, E. Comercio de psitácidos en Nicaragua, del bosque a Managua. Evaluación de la procedencia, métodos de captura y beneficios financieros de la actividad. En publicación: Encuentro Numero 69, Julio-Septiembre de 2004. Managua, Nicaragua: Universidad Centroamericana; 2007.

18. Inskipp, T. \& Gillett, H. J. (Eds.) Checklist of CITES species and Annotated CITES Appendices and reservations. Compiled by UNEPWCMC. Cambridge, UK. CITES Secretariat, Geneva, Switzerland and UNEP-WCMC; 2005.
19. Baptispe M.P., Franco A.M. Especies focales para la conservación en el Departamento de Córdoba. Bogotá D. C., Colombia: Corporación Autónoma Regional de los Valles del Sinú y del San Jorge e Instituto de Recursos Biológicos Alexander Von Humboldt; 2006.

20. Rodriguez-Mahecha J, Rojas F, Arzuza D y González-Hernández A. Loros, Pericos \& Guacamayas Neotropicales. Serie libretas de campo No 2. Bogotá, Colombia: Editorial Panamericana, Formas e Impresos S. A. Conservación Internacional; 2005. 